\title{
On the subsystems of topological Markov chains
}

\author{
WOLFGANG KRIEGER \\ Institut für Angewandte Mathematik der Universität Heidelberg, Im Neuenheimer \\ Feld 294, D-6900, Heidelberg 1
}

(Received 30 October 1981)

\begin{abstract}
Let $S_{\boldsymbol{A}}$ be an irreducible and aperiodic topological Markov chain. If $\boldsymbol{S}_{\bar{A}}$ is an irreducible and aperiodic topological Markov chain, whose topological entropy is less than that of $S_{A}$, then there exists an irreducible and aperiodic topological Markov chain, whose topological entropy equals the topological entropy at $S_{\bar{A}}$, and that is a subsystem of $S_{A}$. If $\bar{S}$ is an expansive homeomorphism of the Cantor discontinuum, whose topological entropy is less than that of $S_{A}$, and such that for every $j \in \mathbb{N}$ the number of periodic points of least period $j$ of $\bar{S}$ is less than or equal to the number of periodic points of least period $j$ of $S_{A}$, then $\bar{S}$ is topological conjugate to a subsystem of $S_{\mathrm{A}}$.
\end{abstract}

Consider an irreducible and aperiodic topological Markov chain. Represent this chain as the shift $S_{A}$ on a shift space $X_{A}$ given by a transition matrix $A$ over a finite state space $\Sigma$,

$$
\begin{gathered}
A\left(\sigma, \sigma^{\prime}\right) \in\{0,1\}, \sigma, \sigma^{\prime} \in \Sigma, \\
X_{A}=\left\{\left(x_{i}\right)_{i \in Z} \in \Sigma^{Z}: A\left(x_{i}, x_{i+1}\right)=1, i \in \mathbb{Z}\right\}, \\
S_{A} x=\left(x_{i+1}\right)_{i \in Z} \quad\left(x=\left(x_{i}\right)_{i \in Z} \in X_{A}\right) .
\end{gathered}
$$

We use the notation

$$
Z(a)=\left\{x \in \Sigma^{Z}: x_{i}=a_{i}, 0 \leq i<I\right\}, \quad a \in \Sigma^{[0, I)}, I \in \mathbb{N},
$$

and

$$
\mathscr{A}\left[S_{\mathrm{A}}, I\right]=\left\{a \in \Sigma^{[0, I)}: A\left(a_{i}, a_{i+1}\right)=1,0 \leq i<I\right\}, \quad I \in \mathbb{N}
$$

and, more generally, given a subshift $(X, S)$ of $\Sigma^{\mathbf{Z}}$, we denote

$$
\mathscr{A}[S, I]=\left\{a \in \Sigma^{[0, I)}: X \cap Z(a) \neq \varnothing\right\}, \quad I \in \mathbb{N} .
$$

Given another irreducible and aperiodic topological Markov chain $S_{\bar{A}}$ such that the entropy condition

$$
h\left(S_{A}\right)>h\left(S_{\bar{A}}\right)
$$

is satisfied, the question arises if $S_{\bar{A}}$ is topologically conjugate to the restriction of $S_{A}$ to a closed $S_{A}$-invariant subset of $X_{A}$. The answer is here not always affirmative, since the presence in $S_{\bar{A}}$ of periodic points of low period can be an obstruction to isomorphically imbedding $S_{\bar{A}}$ into $S_{A}$. In fact, as we shall see in theorem 3 , where we characterize the subsystems of $S_{A}$, this is the only obstruction. However, it is 
always possible, as we shall see in theorems 1 and 2 , to find an irreducible and aperiodic topological Markov chain that is a subsystem of $S_{A}$ and has topological entropy equal to $h\left(S_{\bar{A}}\right)$. The existence of such a chain is significant in connection with certain problems of noiseless coding theory, that deal with a situation where digital data to be recorded or transmitted is to be encoded so as to conform to the design of the device used. (Problems that arise in such a context are e.g. described in $[3, \S \S I V, V]$.) From the existence of such a chain and a theorem of Adler \& Marcus [1] it follows that $S_{\bar{A}}$ can be almost homeomorphically imbedded into $S_{A}$.

The proofs require a few preparations. First we choose $\alpha, \beta, \omega \in \Sigma, \alpha \neq \beta, \alpha \neq \omega$,

$$
A(\beta, \alpha)=A(\beta, \omega)=1,
$$

together with a block

$$
a=\left(a_{i}\right)_{0 \leq i<N} \in \mathscr{A}\left[S_{A}, N\right]
$$

where

$$
a_{0}=\alpha, \quad a_{N-1}=\beta,
$$

and where the symbol $\beta$ appears only once in $a$. Further, let $\gamma \in \Sigma, A(\omega, \gamma)=1$. Let $S_{A, M}$ be the subshift of finite type that arises by excluding from the system $\left(X_{A}, S_{A}\right)$ the block $a^{(*) M}$ (* stands for concatenation). Denote

$$
\mathscr{C}_{M}[I]=\left\{c \in \mathscr{A}\left[S_{A, M}, I\right]: c_{0}=\gamma, c_{I-1}=\beta\right\} .
$$

LEMMA 1. For every $\delta>0$ there are $M, I \in \mathbb{N}$ such that

$$
\left|\mathscr{C}_{M}\left[I^{\prime}\right]\right|>e^{\left(h\left(S_{A}\right)-\delta\right) I^{\prime}}, \quad I^{\prime}>I .
$$

Proof. Let $L$ be such that

$$
\log A^{L-2}\left(\sigma, \sigma^{\prime}\right)>\left(h\left(S_{A}\right)-\frac{\delta}{2}\right) L, \quad \sigma, \sigma^{\prime} \in \Sigma
$$

and let $M$ be such that

$$
N M>L
$$

One has $\mathscr{C}_{M}\left[I^{\prime}\right] \supset \mathscr{B}\left[I^{\prime}\right]$, where

$$
\mathscr{B}\left[I^{\prime}\right]=\left\{\left(b_{i}\right)_{0 \leq i<I^{\prime}} \in \mathscr{A}\left[S_{A^{\prime}} I^{\prime}\right]: b_{0}=\gamma, b_{I^{\prime}-1}=\beta, b_{k L-1}=\beta, b_{k L}=\omega, 0 \leqq k<I^{\prime} L^{-1}\right\} .
$$

If

$$
I^{\prime}>2 L h\left(S_{A}\right) \delta^{-1}
$$

then

$$
\left|\mathscr{B}\left[I^{\prime}\right]\right|>e^{\left(h\left(S_{\mathrm{A}}\right)-\delta\right) I^{\prime}} .
$$

We first turn our attention to an irreducible and aperiodic topological Markov chain $S_{\bar{A}}$ where $h\left(S_{A}\right)>h\left(S_{\bar{A}}\right)$. Let $\bar{A}$ be over a state space $\bar{\Sigma}$, and choose (going to a two-block system if necessary) a $\bar{\rho} \in \bar{\Sigma}$ such that $\bar{A}(\bar{\rho}, \bar{\rho})=0$. For $I \in \mathbb{N}$, where $\bar{A}^{I}$ has all entries positive, we are going to describe an irreducible and aperiodic subshift of finite type $T_{\bar{A}, I}$ as the restriction of the shift to a shift-invariant closed subset $Y_{\tilde{A}, I}$ of the shift space

$$
((\bar{\Sigma}-\{\bar{\rho}\}) \times\{1\}) \cup(\bar{\Sigma} \times\{0\})^{\mathbb{Z}} .
$$


We stipulate that only those two-blocks $\left((\bar{\sigma}, \tau),\left(\bar{\sigma}^{\prime}, \tau^{\prime}\right)\right)$ appear as subblocks of sequences in $Y_{\bar{A}, I}$ where $\bar{A}\left(\bar{\sigma}, \bar{\sigma}^{\prime}\right)=1$, and we stipulate that all $(I+2)$-blocks $\left(c_{i}\right)_{0 \leq i \leq I+1}$ that appear as subblocks of the sequences in $Y_{\bar{A}, I}$ conform to one of the following eight specifications. These are designed to ensure the irreducibility and aperiodicity of $T_{\bar{A}, I}$ and also to ensure that the set

$$
\bar{F}=\left\{y \in Y_{\bar{A}, I}: y_{0} \in(\bar{\Sigma}-\{\bar{\rho}\}) \times\{1\}\right\}
$$

satisfies

$$
\bar{F} \cap T_{\bar{A}, I}^{i} \bar{F}=\varnothing, \quad 0<i<I,
$$

and

$$
Y_{\bar{A}, I}=\bigcup_{0 \leqq i<I} T_{\bar{A}, I}^{i} \bar{F} .
$$

(1) $c_{0} \in(\bar{\Sigma}-\{\bar{\rho}\}) \times\{1\}$,

$c_{i} \in \bar{\Sigma} \times\{0\}, \quad 0<i<I$,

$c_{I} \in(\bar{\Sigma}-\{\bar{\rho}\}) \times\{1\}$,

$c_{I+1} \in \bar{\Sigma} \times\{0\}$.

(2) $c_{0} \in(\bar{\Sigma}-\{\bar{\rho}\}) \times\{1\}$,

$c_{i} \in \bar{\Sigma} \times\{0\}, \quad 0<i<I$,

$c_{I}=(\bar{\rho}, 0)$,

$c_{I+1} \in(\bar{\Sigma}-\{\bar{\rho}\}) \times\{1\}$.

(3) $c_{0}=(\bar{\rho}, 0)$,

$c_{1}=(\bar{\Sigma}-\{\bar{\rho}\}) \times\{1\}$,

$c_{i} \in \bar{\Sigma} \times\{0\}, \quad 1<i \leq I$,

$c_{I+1} \in(\overline{\bar{\Sigma}}-\{\bar{\rho}\}) \times\{1\}$.

(4) $c_{0}=(\bar{\rho}, 0)$,

$c_{1}=(\bar{\Sigma}-\{\bar{\rho}\}) \times\{1\}$,

$c_{i} \in \bar{\Sigma} \times\{0\}, \quad 1<i \leq I$,

$c_{I+1}=(\bar{\rho}, 0)$.

(5) There is a $j, 1<j<I$, such that

$c_{i} \in \bar{\Sigma} \times\{0\}, \quad 0 \leq i<j-1$,

$c_{j-1}=(\bar{\rho}, 0)$,

$c_{j} \in(\bar{\Sigma}-\{\bar{\rho}\}) \times\{1\}$,

$c_{i} \in \bar{\Sigma} \times\{0\}, \quad j<i \leq I+1$.

(6) There is a $\bar{\sigma} \in \bar{\Sigma}-\{\bar{\rho}\}$ such that

$$
\begin{aligned}
& c_{0}=(\bar{\sigma}, 0), \sum_{\bar{\alpha} \neq \bar{\rho}} \bar{A}^{I-1}(\bar{\alpha}, \bar{\sigma})>0, \\
& c_{1} \in(\bar{\Sigma}-\{\bar{\rho}\}) \times\{1\}, \\
& c_{i} \in \bar{\Sigma} \times\{0\}, \quad 1<i \leq I, \\
& c_{I+1} \in(\bar{\Sigma}-\{\bar{\rho}\}) \times\{1\} .
\end{aligned}
$$


(7) There is a $\bar{\sigma} \in \bar{\Sigma}-\{\bar{\rho}\}$ such that

$$
\begin{aligned}
& c_{0}=(\bar{\sigma}, 0), \sum_{\bar{\alpha} \neq \bar{\rho}} \bar{A}^{I-1}(\bar{\alpha}, \bar{\sigma})>0, \\
& c_{1} \in(\bar{\Sigma}-\{\bar{\rho}\}) \times\{1\}, \\
& c_{i} \in \bar{\Sigma} \times\{0\}, \quad 1<i \leq I, \\
& c_{I+1}=(\bar{\rho}, 0) .
\end{aligned}
$$

(8) There is a $j, 1 \leq j \leq I$, and a $\bar{\sigma} \in \bar{\Sigma}$ such that

$$
\begin{aligned}
& c_{0}=(\bar{\sigma}, 0), \sum_{\bar{\alpha} \neq \bar{\rho}} A^{I-j+1}(\bar{\alpha}, \bar{\sigma})>0 \\
& c_{i} \in \bar{\Sigma} \times\{0\}, \quad 1<i<j-1, \\
& c_{j-1} \in(\bar{\Sigma}-\{\bar{\rho}\}) \times\{0\}, \\
& c_{j} \in(\bar{\Sigma}-\{\bar{\rho}\}) \times\{1\}, \\
& c_{i} \in \bar{\Sigma} \times\{0\}, \quad j<i \leq I+1 .
\end{aligned}
$$

THEOREM 1. The topological entropy of $T_{\bar{A}, I}$ equals the topological entropy of $S_{\bar{A}}$. Proof. Let $I^{\prime} \geq I+2$. An inspection of the above eight rules shows that for every

$$
\left(\bar{\sigma}_{i}\right)_{0 \leq i<I^{\prime}} \in \mathscr{A}\left[S_{\bar{A}}, I^{\prime}\right]
$$

there is at least one

$$
\left(\bar{\sigma}_{i}, \tau_{i}\right)_{0 \leq i<I^{\prime}} \in \mathscr{A}\left[T_{\bar{A}, l}, I^{\prime}\right]
$$

and also, that there are at most $I+1$ such elements of $\mathscr{A}\left[T_{\bar{A}, I}, I^{\prime}\right]$.

Remark that the proof of this theorem can also be formulated by saying that the mapping that drops the second components is an at most $I+1$ to 1 continuous mapping of $Y_{\bar{A}, I}$ onto $X_{\bar{A}}$.

Our next theorem can be obtained as a corollary of theorems 1 and 3 . In presenting the following proof, we hope to illustrate the method that is again to be used in the proof of theorem 3.

THEOREM 2. For I sufficiently large, $T_{\bar{A}, I}$ is topologically conjugate to a subsystem of $S_{A}$.

Proof. By lemma 1 we can find $M, I \in \mathbb{N}$ such that

$$
\left|\mathscr{C}_{M}\left[I^{\prime}-N M-1\right]\right|>\left|\mathscr{A}\left[S_{\bar{A}}, I^{\prime}\right]\right|, \quad I^{\prime} \geq I .
$$

We can then have one-to-one into mappings

$$
\phi_{I}: \mathscr{A}\left[S_{\bar{A}}, I\right] \rightarrow \mathscr{C}_{M}[I-N M-1],
$$

and

$$
\phi_{I+1}: \mathscr{A}\left[S_{\bar{A}}, I+1\right] \rightarrow \mathscr{C}_{M}[I-N M]
$$

We form the block

$$
r=a^{(*) M} *(\omega)
$$

Observe that $r$ cannot overlap itself properly, and define a one-to-one continuous and shift-invariant mapping $\Phi$ of $Y_{\bar{A}, I}$ into $X_{A}$ where $\Phi$ carries a $\bar{y} \in Y_{\bar{A}, I}$ into the 
$x \in X_{A}$ that is determined by requiring

$$
\begin{gathered}
\left(x_{i}\right)_{0 \leq i<I}=r * \phi_{I}\left(\left(\bar{\sigma}_{i}\right)_{0 \leq i<I}\right), \\
\bar{y}_{i}=\left(\bar{\sigma}_{i}, \tau_{i}\right), \quad 0 \leq i<I \\
\bar{y}_{0}, \bar{y}_{I} \in(\bar{\Sigma}-\{\bar{\rho}\}) \times\{1\},
\end{gathered}
$$

and

$$
\begin{gathered}
\left(x_{i}\right)_{0 \leq i \leq I}=r * \phi_{I+1}\left(\left(\bar{\sigma}_{i}\right)_{0 \leq i \leq I}\right), \\
\bar{y}_{i}=\left(\bar{\sigma}_{i}, \tau_{i}\right), \quad 0 \leq i \leq I, \\
\bar{y}_{0}, \bar{y}_{I+1} \in(\bar{\Sigma}-\{\bar{\rho}\}) \times\{1\} .
\end{gathered}
$$

We now turn our attention to an expansive homeomorphism $S$ of the Cantor discontinuum. We assume $S$ given as a subshift $(X, S)$ of some full shift space, and we denote by $\mathcal{N}_{S}[J, K], K \geq J$, the set of all $a \in \mathscr{A}[S, K]$ such that there is for every $j, 0<j<J$, at least one $k, 0 \leq k<K-j$ such that

$$
a_{k} \neq a_{k+j} \text {. }
$$

We have the following lemma of Kakutani-Rohlin type.

LEMMA 2. For all $J, K \in \mathbb{N}, K \geq J$, there is a closed open set $F \subset X$ such that

$$
F \cap S^{j} F=\varnothing, \quad 0<j<J
$$

and

$$
\bigcup_{-J<j<J} S^{j} F \supset \bigcup_{a \in \mathcal{N}_{S}[J, K]} X \cap Z(a)
$$

Proof. Enumerate

$$
\left\{Z(a): a \in \mathcal{N}_{s}[J, K]\right\}=\left\{C_{l}: 1 \leqq l \leqq L\right\},
$$

and then obtain inductively an increasing sequence $F_{l}, 1 \leq l \leq L$, of closed open sets by

$$
\begin{gathered}
F_{1}=C_{1} \\
F_{l+1}=F_{l} \cup\left(C_{l+1} \cap\left(X-\bigcup_{-J<j<J} S^{j} F_{l}\right)\right) \quad 1<l<L .
\end{gathered}
$$

Set $F=F_{L}$.

In the sequel $\Pi_{j}$ will indicate the set of periodic points of a homeomorphism that has least period $j$.

THEOREM 3. Let $S$ be an irreducible and aperiodic topological Markov chain, and let $\bar{S}$ be an expansive homeomorphism of the Cantor discontinuum such that

$$
h(S)>h(\bar{S})
$$

and

$$
\left|\Pi_{j}(S)\right| \geq\left|\Pi_{j}(\bar{S})\right|, \quad j \in \mathbb{N},
$$

Then $\bar{S}$ is topologically conjugate to a subsystem of $S$.

Proof. We let $\bar{S}$ be given as a subshift of some shift space $\bar{X}$, and we continue to let the topological Markov chain to be given as $S_{A}$. In view of lemma 1 we have 
an $M \in \mathbb{N}$ such that

$$
h\left(S_{A, M}\right)>h(\bar{S}) .
$$

There is then an $L \in \mathbb{N}$ such that

$$
\left|\Pi_{j}\left(\boldsymbol{S}_{\mathrm{A}, \boldsymbol{M}}\right)\right| \geq\left|\Pi_{j}(\overline{\boldsymbol{S}})\right|, \quad j>L .
$$

Let now $P, Q \in \mathbb{N}$ be such that all entries of $A^{N P}$ are positive, and such that

$$
Q>P+L+M
$$

and form the blocks

$$
\begin{aligned}
& s=a^{(*) P} *(\omega), \\
& t=a^{(*) Q} *(\omega) .
\end{aligned}
$$

By lemma 1, and by (2), there is a $J \in \mathbb{N}, J>(2 P+Q) N+3$ such that

$$
\left|\mathscr{C}_{M}\left[J^{\prime}-(2 P+Q) N-3\right]\right|>\left|\mathscr{A}\left[\bar{S}, J^{\prime}\right]\right|, \quad J^{\prime} \geq J \text {. }
$$

Denote

$$
\begin{array}{ll}
\mathscr{P}_{j}=\left\{b \in \mathscr{A}\left[S_{A}, j\right]: Z(b) \cap \Pi_{j}\left(S_{A}\right) \neq \varnothing\right\}, & 0<j<L, \\
\mathscr{P}_{j}=\left\{b \in \mathscr{A}\left[S_{A, M}, j\right]: Z(b) \cap \Pi_{j}\left(S_{A, M}\right) \neq \varnothing\right\}, & L \leq j<J, \\
\overline{\mathscr{P}}_{j}=\left\{\bar{b} \in \mathscr{A}[\bar{S}, j]: Z(\bar{b}) \cap \Pi_{j}(\bar{S}) \neq \varnothing\right\}, & 0<j<J .
\end{array}
$$

By (1) and (3) one can assign in a one-to-one manner to every $\bar{b} \in \overline{\mathscr{P}}_{j}$ a $b \in \mathscr{P}_{j}$, $0<j<J$, such that, if

$$
\left(\bar{b}_{0}, \bar{b}_{1}, \ldots, \bar{b}_{j-1}\right) \rightarrow\left(b_{0}, b_{1}, \ldots, b_{j-1}\right),
$$

then

$$
\left(\bar{b}_{j-1}, \bar{b}_{0}, \ldots, \bar{b}_{j-2}\right) \rightarrow\left(b_{j-1}, b_{0}, \ldots, b_{j-2}\right), \quad 0<j<J .
$$

By (5) one can also have one-to-one into mappings

$$
\psi_{j}: \mathscr{A}[\bar{S}, j] \rightarrow \mathscr{C}_{M}[j-(2 P+Q) N-3], \quad J \leq j<2 J .
$$

Finally we select for all $\bar{b} \in \bar{P}_{j}, 0<j<J$, blocks

$$
s_{-}(b), s_{+}(b) \in \mathscr{A}\left[S_{A}, N P+1\right]
$$

such that

$$
\begin{gathered}
\left(s_{-}(b)\right)_{N P}=\beta, \quad\left(s_{+}(b)\right)_{0}=\gamma, \\
A\left(\bar{b}_{j-1}, s_{-}(b)_{0}\right)=A\left(\left(s_{+}(b)\right)_{N P}, \bar{b}_{0}\right)=1 .
\end{gathered}
$$

There is a $K \in \mathbb{N}, K>J^{2}$, such that

$$
\left|\mathscr{A}[\bar{S}, K]-\mathcal{N}_{\bar{S}}[J, K]\right|=\sum_{0<j \leq J}\left|\Pi_{j}(\overline{\mathcal{S}})\right|
$$

for if no such $K$ existed, then it would follow by a compactness argument that for some $j, 0<j \leq J, \bar{S}$ had more than $\left|\Pi_{j}(\tilde{S})\right|$ periodic points of least period $j$. We apply now lemma 3 to obtain a closed open set $F \subset \bar{X}$ such that

$$
\begin{gathered}
F \cap S^{i} F=\varnothing, \quad 0<i<J, \\
\bigcup_{-J<i<J} S^{i} F \supset \bigcup_{\bar{d} \in \mathcal{N}_{S}[J, K]} \bar{X} \cap Z(\bar{d}) .
\end{gathered}
$$


Define then an $\tilde{F}$ by setting

$$
\begin{gathered}
F_{-}=\left(\bigcap_{-2 J<i<0}\left(\bar{X}-\bar{S}^{i} F\right)\right) \cap F, \\
F_{+}=F \cap\left(\bigcap_{0<i<2 J}\left(\bar{X}-S^{-i} F\right)\right) \\
\tilde{F}=\bar{S}^{J} F_{-} \cup F \cup S^{-J} F,
\end{gathered}
$$

and have

$$
\begin{gathered}
\tilde{F} \cap \bar{S}^{i} \tilde{F}=\varnothing, \quad 0<i<J, \\
\bigcap_{-J<i<J}\left(\bar{X}-S^{i} \tilde{F}\right) \subset \bigcup_{0<j<J} \bigcup_{\bar{d} \in \Phi_{j}-J<i<J} \bigcap_{\bar{X}} \cap S^{i} Z(\bar{d}) .
\end{gathered}
$$

Thus one can define a continuous shift invariant mapping $\Psi$ of $\bar{X}$ into $X_{A}$ where $\Psi$ carries an $\bar{x} \in \bar{X}$ into the $x \in X_{A}$ that is determined by the following rules:

(1) If

$$
\bar{x} \in Z(\bar{b}) \cap \bigcap_{-J<i<J}(\bar{X}-\bar{S} i \tilde{F}), \quad \bar{b} \in \overline{\mathscr{P}}_{i}, \quad 0<j<J
$$

then

$$
\left(x_{i}\right)_{0 \leq i<j}=b \text {. }
$$

(2) If

$$
\begin{gathered}
\overline{\boldsymbol{X}} \in\left(\bigcap_{-2 J<i<0}\left(\bar{X}-\overline{S^{i}} \tilde{F}\right)\right) \cap S^{i} Z(\bar{b}) \cap \tilde{F} \cap\left(\bigcap_{0<i<I_{+}}\left(\bar{X}-\bar{S}^{-i} \tilde{F}\right)\right) \cap \bar{S}^{I^{+}} \tilde{F}, \\
J<I_{+}<2 J, \quad \bar{b} \in \overline{\mathscr{P}}_{i}, \quad 0<j \leq J,
\end{gathered}
$$

then

$$
\left(x_{i}\right)_{-\left[(2 J-1) j^{-1}\right] j \leq i<I_{+}}=b^{(*)[2 J-1] j^{-1}} * s_{-}(b) * t * s * \psi\left(\left(\bar{x}_{i}\right)_{0 \leq i<I_{+}}\right) .
$$

(3) If

$$
\begin{gathered}
\bar{x} \in S^{I}-\tilde{F}\left(\bigcap_{-I<i<0}\left(\bar{X}-\bar{S}^{i} \tilde{F}\right)\right) \cap \tilde{F} \cap Z(b) \cap\left(\bigcap_{0<i<2 J}\left(\bar{X}-\bar{S}^{-i} \tilde{F}\right)\right), \\
J<I_{-}<2 J, \quad \bar{b} \in \overline{\mathscr{P}}_{i}, \quad 0<j \leq J,
\end{gathered}
$$

then

$$
\left(x_{i}\right)_{-I_{-}<i \leq\left[(2 J-1) i^{-1}\right] j}=\psi\left(\left(\bar{x}_{i}\right)_{-I_{-}<i \leq 0}\right) * s * t * s_{+}(b) * b^{(*)[2 J-1] j^{-1}} .
$$

(4) If

$$
\begin{gathered}
\bar{x} \in S^{I_{-}} \tilde{F} \cap\left(\bigcap_{-I_{-}<i<0}(X-S i \tilde{F})\right) \cap \tilde{F} \cap\left(\bigcap_{0<i<I_{+}}\left(\bar{X} \cap \bar{S}^{-i} \tilde{F}\right)\right) \cap \bar{S}^{I_{+}} \tilde{F}, \\
J<I_{-}, \quad I_{+}<2 J,
\end{gathered}
$$

then

$$
\left(x_{i}\right)_{-I_{-}+L^{\prime} \leq i \leq I_{+}}=\psi\left(\left(\bar{x}_{i}\right)_{-I_{-} \leq i<0}\right) * s * t * s * \psi\left(\left(\bar{x}_{i}\right)_{0 \leq i<I_{+}}\right) .
$$

The structure of the blocks $s$ and $t$ and (4) show that $x \in F$ if and only if

$$
\Psi x \in S_{A}^{(2 P+Q) N+3} Z(t) .
$$


Since we have (6) and since the assignments that entered into the construction of $\Psi$ are one-to-one we conclude that $\Psi \bar{x}$ uniquely determines $\bar{x}$.

There is a version of the finite generator theorem for ergodic measure preserving transformations of finite entropy, that realizes such a transformation by means of an invariant probability measure of any irreducible and aperiodic topological Markov chain, whose topological entropy exceeds the entropy of the transformation ([4], [2 § 28]). One can say that a corollary of theorem 3 achieves for minimal expansive homeomorphisms of the Cantor discontinuum what the finite generator theorem does for measure preserving transformations.

Corollary. Let $\mathrm{S}$ be an irreducible and aperiodic topological Markov chain and let $\bar{S}$ be a minimal expansive homeomorphism of the Cantor discontinuum such that

$$
h(S)>h(\bar{S}) \text {. }
$$

Then $\bar{S}$ is topologically conjugate to a subsystem of $S_{A}$.

My thanks go to Roy Adler and Brian Marcus for their stimulating questions and comments. This work was done under the auspices of Deutsche Forschungsgemeinschaft at the 'Sonderforschungsbereich Stochastische Mathematische Modelle' at the University of Heidelberg.

\section{REFERENCES}

[1] R. Adler \& B. Marcus. Topological entropy and equivalence of dynamical systems. Mem. Amer. Math. Soc. 219 (1979).

[2] M. Denker, Ch. Grillenberger \& K. Sigmund. Ergodic Theory on Compact Spaces. Lecture Notes in Math. No. 527. Springer: Berlin, 1976.

[3] H. Kobayashi. A survey of coding schemes for transmission and recording of digital data. IEEE Trans. Comm. 19 (1971), 1087-1100.

[4] W. Krieger. On generators in ergodic theory. Proceedings of the International Congress of Mathematicians. Vancouver 1974, Vol. 2, pp. 303-308. 